\section{Finite-Elemente-Analyse zur Berechnung einer Osteosynthese am posterioren Malleolus}

Anwar et al. Finite element analysis of the three different posterior malleolus fixation strategies in relation to different fracture sizes. Injury $2017 ; 48$ : 825-832

Die optimale Osteosynthese bei Frakturen des posterioren Malleolus bleibt aktuell unklar, da der Zugang sowie die Wahl des jeweiligen Implantats von der Größe des frakturierten Fragmentes abhängig ist. Ziel der hier vorgestellten Studie war es zu berechnen, wie 3 unterschiedliche osteosynthetische Versorgungen des dorsalen Malleolus die Stabilität der Fraktur und der Osteosynthese beeinflussen.

Für diese Studie wurde anhand von gesunden humanen CT-Rohdaten die distale Tibia digital isoliert, rekonstruiert und eine Fraktur des dorsalen Malleolus kalkuliert. Die Fraktur des dorsalen Malleolus betrug 30, 40 oder $50 \%$ der distalen tibialen Gelenkfläche. Anschließend wurde digital eine Osteosynthese im Modell eingepflegt mittels dreier unterschiedlicher Osteosyntheseverfahren: einer indirekten anterioren oder direkten Zugschraubenosteosynthese von posterior bzw. einer dorsalen Plattenosteosynthese. Die Simulation wurde mit einer axialen Last an der Tibia von 350 oder $700 \mathrm{~N}$ durchgeführt zur Simulation eines Zweibein- bzw. eines Einbeinstands. Bei der Modellierung wurde die distale Tibia fixiert und erhielt hierfür 0 Freiheitsgrade.

Die relative Fragmentdislokation nach osteosynthetischer Versorgung zeigte sich in der Gruppe der dorsalen Plattenosteosynthese im Vergleich zu den Gruppen der Schraubenosteosynthese fast um das 10-Fache reduziert. Dieses Ergebnis ließ sich bei allen Frakturtypen (30, 40 und $50 \%$ ) als auch bei allen axialen Lastsimulationen (350 und $700 \mathrm{~N}$ ) widerspiegeln. Die Analyse der Von-Mises-Spannungsverteilung an den Implantaten zeigte, dass die dorsale Plattenostesynthese signifikant höherem Stress im Vergleich zu p.-a. oder a.-p. Schraubenosteosynthesen aus- gesetzt ist. Dieser Stress verteilt sich wiederum gleichmäßig am gesamten Implantat, ohne potenzielle Sollbruchstellen zu erzeugen. Im Gegensatz dazu sind a.-p. oder p.-a. Schraubenosteosynthesen lokalem Stress ausgesetzt. Besonders betroffen davon scheint die mediale Schraube zu sein. Die zuvor erwähnten Ergebnisse wurden statistisch signifikant bestätigt durch eine selektive Knotenpunktanalyse. Eine zusätzliche negative Korrelationsanalyse zwischen Implantatstress und Fragmentdislokation impliziert, dass p.-a. oder a.-p. Schraubenosteosynthesen eine weniger stabile Fixierung als die Platte darstellen.

\section{FAZIT}

Aus kalkulatorischer Sicht bietet eine „buttress“-Platte im Sinne einer posterioren Osteosynthese des dorsalen Malleolus eine höhere Stabilität im Vergleich zu einer direkten oder indirekten Verschraubung. Zusätzlich erweist sich die Wahl der dorsalen Plattenosteosynthese bei zunehmender Größe der Fraktur als eine sichere und stabilere Form der internen Fixation. Unterschiede in der Frakturstabilität zwischen einer p.-a. oder a.-p. Schraubenosteosynthese ließen sich nicht nachweisen.

PD Dr. med. Ioannis Stratos, Rostock 